\title{
Kinematic difference between a biological cell and an artificial vesicle in a strong DC electric field - a "shell" membrane model study
}

Hui Ye(D)

\begin{abstract}
Background: Cellular biomechanics can be manipulated by strong electric fields, manifested by the field-induced membrane deformation and migration (galvanotaxis), which significantly impacts normal cellular physiology. Artificial giant vesicles that mimic the phospholipid bilayer of the cell membrane have been used to investigate the membrane biomechanics subjected to electric fields. Under a strong direct current (DC) electric field, the vesicle membrane demonstrates various patterns of deformation, which depends on the conductivity ratio between the medium and the cytoplasm. The vesicle exhibits prolate elongation along the direction of the electric field if the cytoplasm is more conductive than the medium. Conversely, the vesicle exhibits an oblate deformation if the medium is more conductive. Unlike a biological cell, artificial vesicles do not migrate in the strong DC electric field.

To reconcile the kinematic difference between a cell and a vesicle under a strong DC electric field, we proposed a structure that represents a low-conductive, "shell-like" membrane. This membrane separates the extracellular medium from the cytoplasm. We computed the electric field, induced surface charge and mechanical pressure on the fixed membrane surface. We also computed the overall translational forces imposed on the structure for a vesicle and a cell.

Results: The DC electric field generated a steady-state radial pressure due to the interaction between the local electric field and field-induced surface charges. The radial pressure switches its direction from "pulling" to "compressing" when the medium becomes more conductive than the cytoplasm. However, this switch can happen only if the membrane becomes extremely conductive under the strong electric field. The induced surface charges do not contribute to the net translational force imposed on the structure. Instead, the net translational force generated on the shell structure depends on its intrinsic charges. It is zero for the neutrally-charged, artificial vesicle membrane. In contrast, intrinsic charges in a biological cell could generate translational force for its movement in a DC electric field.
\end{abstract}

Conclusions: This work provides insights into factors that affect cellular/vesicle biomechanics inside a strong DC electric field. It provides a quantitative explanation for the distinct kinematics of a spherical cell verses a vesicle inside the field.

Keywords: Direct current (DC) electric field, Cell, Vesicle, Model, Membrane, Biomechanics 


\section{Background}

Cellular biomechanics can be manipulated by strong electric fields. For example, undulation can be induced on a poorly conductive membrane [1] by an electric field. Tension and poration can be generated in the cell membranes by a microelectrode close to a cell [2]. As a consequence, the cell membrane undergoes geometrical changes. Strong direct current (DC) electric fields cause deformation in the cell membrane with predicable patterns [3, 4]. Stem cells derived from human adipose tissue elongated inside a DC electric field, in a direction that is perpendicular to the field [5]. Electric fieldinduced mechanical signals can be transferred into the biological system and cause a diversity of biological responses, including cell proliferation and apoptosis, hypertrophy (increased cell size), extracellular matrix remodeling, and DNA/RNA synthesis [6].

A DC electric field can also cause cell migration, which has significant impact on normal cellular physiology. The first observation that cells can migrate inside an applied electric field, or galvanotaxis, was reported nearly a century ago [7]. It has been suggested that mechanic forces generated on the cell could be partially responsible for cell migration in the electric field [8]. Cell electrophoresis is a method for estimating the surface charge of a cell by looking at its rate of movement in a DC electrical field [9]. It has been widely used for the characterization of the surface properties of the membrane, as well as for the separation of uniform cell subpopulations in a cell mixture [9]. In addition, electric fields are used for axonal guidance [10, 11], and migration of stem cells [12] or neurons within neural networks [13].

To investigate the cellular biomechanics under electric fields, investigators use artificially generated giant vesicles, which mimic the phospholipid bilayer in the cell membrane $[14,15]$. These vesicles are usually formed with neutral molecules including L-a-phosphatidylcholine [15-17].
Similar to biological cells, vesicles deformed in a very strong DC field [16]. Vesicles exposed to the DC electric pulses can be deformed into elliptical [18] or cylindrical shapes [15]. Deformation of the vesicle depends on the "conductivity ratio" between the cytoplasm and the extracellular medium. The vesicle elongates in the field direction along its axis (prolate) if the cytoplasmic conductivity is higher than the medium. Conversely, the vesicle shortens (oblate) in the field direction if the medium is more conductive. Theoretical works have been proposed to investigate this interior-to-exterior conductivity ratio-dependent membrane deformation, using analytical $[15,19,20]$ or numerical methods [21-23]. Interestingly, vesicle migration has not been observed in the strong DC electric field $(2.0 \mathrm{Kv} / \mathrm{cm})$, despite the observed electroporation and pore formation on the membrane [16].

We use a modeling approach to reconcile the kinematic difference between a biological cell and a vesicle under a DC electric field, with a "shell-like" membrane structure that separates the extracellular medium from the cytoplasm. We analyzed the biomechanics of this 3D structure by computing the steady-state pressure and forces imposed on the fixed membrane surface by the DC electric field. The model confirms the impact of the conductivity ratio on the buildup of the electric pressure on the membrane. The model suggests that the kinematic difference between the vesicle and the biological cell inside a DC electric field can be explained by the lack of charged molecules in the vesicle membrane.

\section{Methods}

\section{"Shell model' of a spherical vesicle/cell in a DC electric} field

Figure 1 illustrates the configuration of a modeled vesicle/cell inside the extracellular medium (0\#). The model contains a spherical membrane shell (1\#) and intracellular cytoplasm (2\#). The center of the sphere is at point $O$. To simplify calculations, we assume that
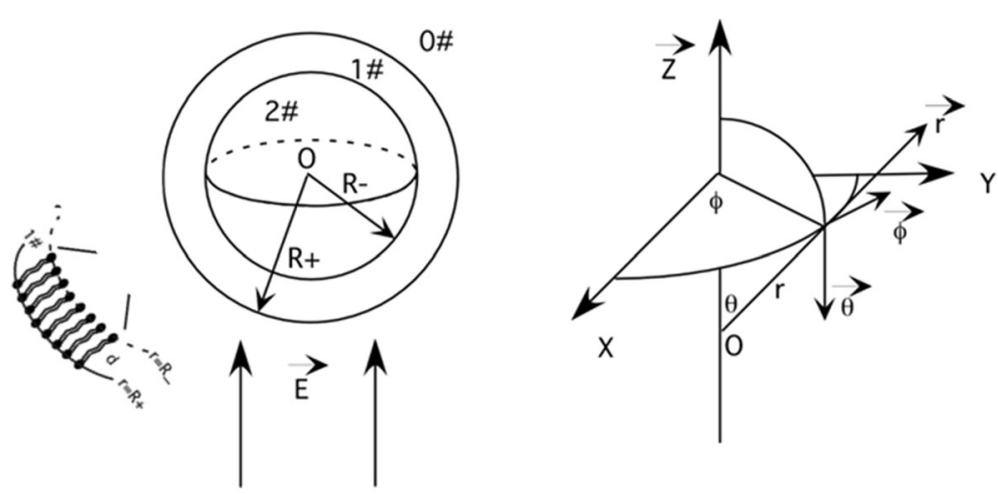

Fig. 1 The spherical vesicle/cell model and the spherical coordinate system $(r, \theta, \varphi)$. The vesicle/cell model includes three homogenous, isotropic regions: the extracellular medium, the membrane, and the cytoplasm. The axis of the electric field overlaps with the $O Z$ axis 
each modeled region is electrically homogenous and isotropic. The dielectric permittivities and the conductivities in the three regions are $\varepsilon_{0}, \varepsilon_{1}, \varepsilon_{2}$ and $\sigma_{0}, \sigma_{1}, \sigma_{2}$, respectively. The inner and outer radii of the membrane are $R_{-}$and $R_{+}$, respectively. The membrane thickness is $d=R_{+}-R_{-}$. The cell is exposed to a uniform external field $\vec{E}=E \vec{Z}$, where $\vec{Z}$ is the unitary vector in the direction of the external field. Similar model configurations have been used to study the bioelectricity of cells under electromagnetic field exposure [24-26].

\section{Model parameters}

Table 1 summarizes the parameters for the model, which are chosen from previous publications [27, 28]. These parameters include geometrical and electrical parameters of the cell and the medium. The electric field intensity was $200,000 \mathrm{~V} / \mathrm{m}$, sufficient to deform the cell membrane $[15,16]$.

\section{Equations and boundary conditions}

Expression of electric potentials in the extracellular medium, the membrane, and the cytoplasm are obtained by solving Laplace's equation [29] with appropriate boundary conditions

$$
\nabla^{2} V=0
$$

Several boundary conditions are considered in the model: (1) Across the boundary of two different media, electric potential is continuous; (2) Across the boundary of two different media, the normal component of the current density is continuous. To account for the dielectric permittivity of the material, "complex conductivity", defined as $S=\sigma+j \omega \varepsilon$, was used [26, 30]. $\omega$ is the angular frequency. It is zero for the DC electric field. $j=\sqrt{-1}$ is the imaginary unit. Therefore, across the extracellular medium/membrane interface (0\#1\#),

$$
S_{0} E_{0 r}-S_{1} E_{1 r}=0
$$

Across the membrane/cytoplasm interface (1\#2\#),

$$
S_{1} E_{1 r}-S_{2} E_{2 r}=0
$$

where $S_{0}=\sigma_{0}+j \omega \varepsilon_{0}, S_{1}=\sigma_{1}+j \omega \varepsilon_{1}, S_{2}=\sigma_{2}+j \omega \varepsilon_{2}$. Presence of the vesicle/cell in the electric field does not perturb field distribution at an infinite distance; and (4), the electric potential inside the vesicle/cell is finite.

In spherical coordinates $(r, \theta, \phi)$, expressions of electric fields are given by

$$
\vec{E}=-\nabla V=-\left(\frac{\partial V}{\partial r}, \frac{1}{r} \frac{\partial V}{\partial \theta}, \frac{1}{r \sin \theta} \frac{\partial V}{\partial \phi}\right)
$$

\section{Surface charge distribution and mechanical pressure on the "shell"}

Under electromagnetic exposure, free charges accumulate on the interface of the two inhomogeneous media [31]. At the $0 \# 1 \#$ interface $\left(r=R_{+}\right)$, the surface charge density is $\rho_{s 01}=\vec{n} \cdot\left(\varepsilon_{1} \vec{E}_{1}-\varepsilon_{0} \vec{E}_{0}\right)$ or

$$
\rho_{s 01}(R, \theta, \phi)=\varepsilon_{1} E_{1 r}-\varepsilon_{0} E_{0 r}
$$

At the $1 \# 2 \#$ interface $\left(\mathrm{r}=\mathrm{R}_{-}\right)$, the surface charge density is $\rho_{s 12}=\vec{n} \cdot\left(\varepsilon_{2} \vec{E}_{2}-\varepsilon_{1} \vec{E}_{1}\right)$ or

$$
\rho_{s 12}(R, \theta, \phi)=\varepsilon_{2} E_{2 r}-\varepsilon_{1} E_{1 r},
$$

where $\vec{n}$ denoted the outward unit normal vector.

Interaction between the free charges and the electric field produces electric pressure, whose radial component can be calculated as the product of the charge density and the averaged electric field on both sides of the

\begin{tabular}{|c|c|c|c|}
\hline Parameters & Standard value & Lower limit & Upper limit \\
\hline Extracellular conductivity $\left(\sigma_{0}, \mathrm{~S} / \mathrm{m}\right)$ & 0.3 & 0.01 & 5 \\
\hline Extracellular dielectric permittivity $\left(\varepsilon_{0}\right.$, As/Nm) & $6.4 \times 10^{-10}$ & - & - \\
\hline Membrane conductivity $\left(\sigma_{1}, \mathrm{~S} / \mathrm{m}\right)$ & $1.2 \times 10^{-6}$ & $5 \times 10^{-7}$ & $5.0 \times 10^{-2}$ \\
\hline Cell membrane dielectric permittivity $\left(\varepsilon_{1}\right.$, As $\left./ \mathrm{Nm}\right)$ & $4.4 \times 10^{-11}$ & $1.8 \times 10^{-11}$ & $8.8 \times 10^{-11}$ \\
\hline Cytoplasmic conductivity $\left(\sigma_{1}, \mathrm{~S} / \mathrm{m}\right)$ & 1.2 & - & - \\
\hline Cytoplasmic dielectric permittivity $\left(\varepsilon_{2}\right.$, As $\left./ \mathrm{Nm}\right)$ & $6.4 \times 10^{-10}$ & $3.5 \times 10^{-10}$ & $7.0 \times 10^{-10}$ \\
\hline Cell radius $(R, \mu \mathrm{m})$ & 10 & 1 & 100 \\
\hline Membrane thickness $(d, n m)$ & 5 & 3 & 7 \\
\hline Electric field intensity $(\mathrm{E}, \mathrm{V} / \mathrm{m})$ & 200,000 & - & \\
\hline
\end{tabular}
interface surface [29]. Quantitatively significant pressure produced by high-intensity electric field could cause cell membrane deformation [32]. On the 0\#1\# interface $\left(r=R_{+}\right)$, the radial pressure is

Table 1 Model parameters 


$$
\rho_{r 01}=\frac{1}{2}\left(E_{0 r}+E_{1 r}\right) \rho_{s 01}
$$

On the $1 \# 2 \#$ interface $\left(r=R_{-}\right)$, the radial pressure is

$$
\rho_{r 12}=\frac{1}{2}\left(E_{1 r}+E_{2 r}\right) \rho_{s 12}
$$

Since the membrane is thin and uncompressible, the net radial electric stress is computed as [33]

$$
P_{r}=P_{r 01}+P_{r 12}
$$

\section{Results}

\section{Analytical solutions for the electric fields and surface} charges

Laplace's equation was solved with boundary conditions (1-4) to yield the expression of the electric potentials (Additional file 1). The potential distributions in the three regions are

$V_{0}=\left(\frac{1}{r^{2}} \frac{3 S_{0}\left(2 S_{1}+S_{2}\right) R_{+}^{6}+3 S_{0}\left(S_{1}-S_{2}\right) R_{+}^{3} R_{-}^{3}}{2\left(S_{1}-S_{0}\right)\left(S_{1}-S_{2}\right) R_{-}^{3}-\left(S_{1}+2 S_{0}\right)\left(2 S_{1}+S_{2}\right) R_{+}^{3}}+\frac{R_{+}^{3}}{r^{2}}-r\right) E \cos \theta$

$$
V_{1}=\frac{3 S_{0} R_{+}^{3}\left[r\left(2 S_{1}+S_{2}\right)+\left(S_{1}-S_{2}\right) \frac{R_{-}^{3}}{r^{2}}\right]}{2\left(S_{1}-S_{0}\right)\left(S_{1}-S_{2}\right) R_{-}^{3}-\left(S_{1}+2 S_{0}\right)\left(2 S_{1}+S_{2}\right) R_{+}^{3}} E \cos \theta
$$

$$
V_{2}=\frac{9 S_{0} S_{1} R_{+}^{3} r}{2\left(S_{1}-S_{0}\right)\left(S_{1}-S_{2}\right) R_{-}^{3}-\left(S_{1}+2 S_{0}\right)\left(2 S_{1}+S_{2}\right) R_{+}^{3}} E \cos \theta
$$

We further calculate the electric field distribution in the extracellular space, the shell membrane, and the cytoplasm using Eq. (4). The expressions for the electric field around the cell, in the extracellular medium (0\#) are:

$$
E_{0 r}=\left[1-\frac{2 R_{+}^{3}}{r^{3}} \frac{R_{-}^{3}\left(S_{0}+2 S_{1}\right)\left(S_{1}-S_{2}\right)+R_{+}^{3}\left(S_{0}-S_{1}\right)\left(2 S_{1}+S_{2}\right)}{2 R_{-}^{3}\left(S_{0}-S_{1}\right)\left(S_{1}-S_{2}\right)+R_{+}^{3}\left(2 S_{0}+S_{1}\right)\left(2 S_{1}+S_{2}\right)}\right] E \cos \theta
$$

$$
E_{0 \theta}=-\left[1+\frac{R_{+}^{3}}{r^{3}} \frac{R_{-}^{3}\left(S_{0}+2 S_{1}\right)\left(S_{1}-S_{2}\right)+R_{+}^{3}\left(S_{0}-S_{1}\right)\left(2 S_{1}+S_{2}\right)}{2 R_{-}^{3}\left(S_{0}-S_{1}\right)\left(S_{1}-S_{2}\right)+R_{+}^{3}\left(2 S_{0}+S_{1}\right)\left(2 S_{1}+S_{2}\right)}\right] E \sin \theta
$$

$$
E_{0 \phi}=0
$$

The electric fields inside the membrane (1\#) are:

$$
E_{1 r}=\frac{3 R_{+}^{3}}{r^{3}} \frac{S_{0}\left[2 R_{-}^{3}\left(-S_{1}+S_{2}\right)+r^{3}\left(2 S_{1}+S_{2}\right)\right]}{2 R_{-}^{3}\left(S_{0}-S_{1}\right)\left(S_{1}-S_{2}\right)+R_{+}^{3}\left(2 S_{0}+S_{1}\right)\left(2 S_{1}+S_{2}\right)} E \cos \theta
$$

$E_{1 \theta}=-\frac{3 R_{+}^{3}}{r^{3}} \frac{S_{0}\left[R_{-}^{3}\left(S_{1}-S_{2}\right)+r^{3}\left(2 S_{1}+S_{2}\right)\right]}{2 R_{-}^{3}\left(S_{0}-S_{1}\right)\left(S_{1}-S_{2}\right)+R_{+}^{3}\left(2 S_{0}+S_{1}\right)\left(2 S_{1}+S_{2}\right)} E \sin \theta$

$$
E_{1 \phi}=0
$$

The electric fields inside the cytoplasm (2\#) are:

$$
E_{2 r}=\frac{9 S_{0} S_{1} R_{+}^{3}}{2 R_{-}^{3}\left(S_{0}-S_{1}\right)\left(S_{1}-S_{2}\right)+R_{+}^{3}\left(2 S_{0}+S_{1}\right)\left(2 S_{1}+S_{2}\right)} E \cos \theta
$$

$$
E_{2 \theta}=-\frac{9 S_{0} S_{1} R_{+}^{3}}{2 R_{-}^{3}\left(S_{0}-S_{1}\right)\left(S_{1}-S_{2}\right)+R_{+}^{3}\left(2 S_{0}+S_{1}\right)\left(2 S_{1}+S_{2}\right)} E \sin \theta
$$

$$
E_{1 \phi}=0
$$

From Eq. (5), the electrically-induced charge density on the $0 \# 1 \#$ interface is (Additional file 2)

$\rho_{s 01}(R, \theta, \phi)=-\frac{3\left[2 R_{-}^{3}\left(S_{1}-S_{2}\right)-R_{+}^{3}\left(2 S_{1}+S_{2}\right)\right]}{2 R_{-}^{3}\left(S_{0}-S_{1}\right)\left(S_{1}-S_{2}\right)+R_{+}^{3}\left(2 S_{0}+S_{1}\right)\left(2 S_{1}+S_{2}\right)}\left(S_{1} \varepsilon_{0}-S_{0} \varepsilon_{1}\right) E \cos \theta$

One the $1 \# 2 \#$ interface (Additional file 3 )

$\rho_{s 12}(R, \theta, \phi)=-\frac{9 R_{+}^{3} S_{0}}{2 R_{-}^{3}\left(S_{0}-S_{1}\right)\left(S_{1}-S_{2}\right)+R_{+}^{3}\left(2 S_{0}+S_{1}\right)\left(2 S_{1}+S_{2}\right)}\left(S_{2} \varepsilon_{1}-S_{1} \varepsilon_{2}\right) E \cos \theta$

Figure 2 illustrates the surface charge distributions on these two interfaces, which depend on the orientation of the cell to the electric field (i.e., $\cos \theta$ term). The densities of the induced surface charges are also functions of the electric properties of the medium, the membrane, and the cytoplasm. The two pole areas carry the greatest density of induced charges. Since the $\cos \theta$ term presents in both Eqs. (14.1) and (14.2), charge distribution on both $0 \# 1 \#$ interface (Fig. 2a) and $1 \# 2 \#$ interface (Fig. 2b) are similar. However, charges carried by the two interfaces have different polarities at the same location (latitude and longitude).

Surface charge density is a function of the field intensity. We compute the maximal surface charge (at $\theta=0$ ) with parameters presented in Table $1(\mathrm{E}=200,000 \mathrm{~V} / \mathrm{m}$, $\sigma 0=0.3 \mathrm{~S} / \mathrm{m}$ and $\sigma 2=1.2 \mathrm{~S} / \mathrm{m})$. The induced charge density is $-0.025 \mathrm{C} / \mathrm{m} 2$ on the $0 \# 1 \#$ interface, and is $0.025 \mathrm{C} / \mathrm{m} 2$ on the $1 \# 2 \#$ interface. Those values are comparable to the charge density carried by the intrinsic membrane proteins $[8,34]$.

Since induced charges are restricted on the membrane surface [35], the net induced charge should be zero on both the $0 \# 1 \#$ and $1 \# 2 \#$ interfaces. This could be confirmed by integrating the surface charge density over the whole surface area. 

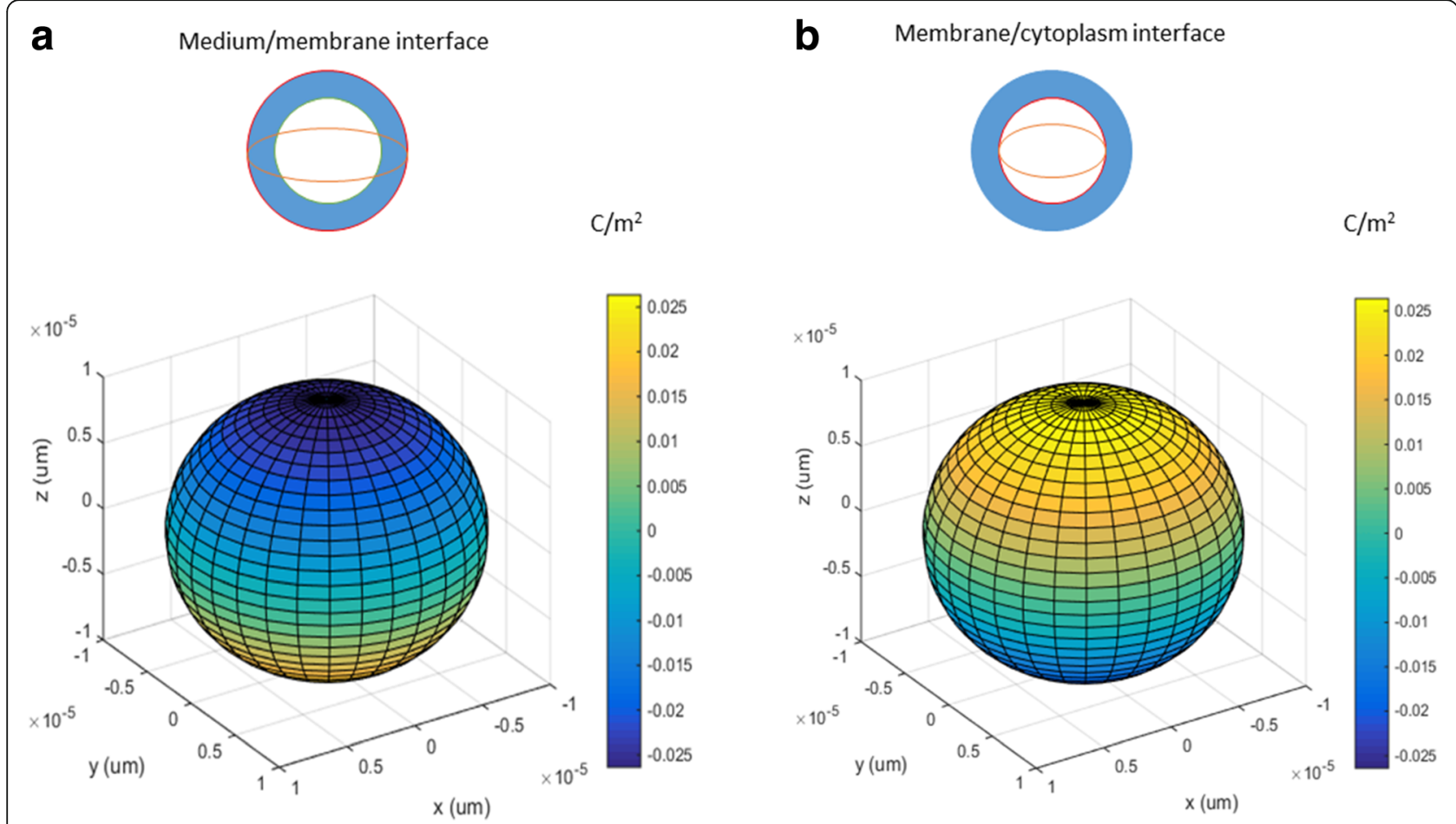

Fig. 2 Electrically-induced surface charge distribution on the a medium/membrane interface ( $\rho$ s01) and $\mathbf{b}$ membrane/cytoplasm interface ( $\rho s 12)$. Membrane thickness is exaggerated to show the inner and outer membrane interfaces. The plot demonstrates the geometrical pattern of the induced surface charge distribution in the DC electric field. The orientation of the shell structure to the field is the same as that shown in Fig. 1. The color represents the amount of the charge density $\left(\mathrm{C} / \mathrm{m}^{2}\right)$ calculated with $\sigma_{0}=0.3 \mathrm{~S} / \mathrm{m}$ and $\sigma_{2}=1.2 \mathrm{~S} / \mathrm{m}$ and $\mathrm{E}=200,000 \mathrm{~V} / \mathrm{m}$

$$
\begin{array}{r}
Q_{s 01}=\iint_{\theta, \phi} \rho_{s 01} d a_{01}=\iint_{\theta, \phi} \rho_{s 01} R_{+}^{2} \sin \theta d \theta d \phi=0 \\
Q_{s 12}=\iint_{\theta, \phi} \rho_{s 12} d a_{12}=\iint_{\theta, \phi} \rho_{s 12} R_{-}^{2} \sin \theta d \theta d \phi=0
\end{array}
$$

Here, $d \widehat{01} \widehat{\widehat{a}}=R_{+}{ }^{2} \sin \theta d \theta d \phi \widehat{r}$ is the surface element on the $0 \# 1 \#$ interface and $d \widehat{a}=R^{2} \sin \theta d \theta d \phi \widehat{r}$ is the surface element on the $12 \#$ interface in the $\widehat{r}$ direction.

\section{Steady state radial pressure}

Mechanical pressure can be generated when the electric field interacts with the induced surface charges [32]. The pressure on the radial $\widehat{r}$ direction is essential for cytoskeletal compression and extension [32]. On the 0\#1\# interface, the steady state radial pressure is (Additional file 2)

$$
P_{r 01}=-\frac{9 E^{2}\left(S_{0}+S_{1}\right)\left[R_{+}{ }^{3}\left(2 S_{1}+S_{2}\right)-2 R^{3}\left(S_{1}-S_{2}\right)\right]^{2}}{2\left[2 R^{3}\left(S_{0}-S_{1}\right)\left(S_{1}-S_{2}\right)+R_{+}{ }^{3}\left(2 S_{0}+S_{1}\right)\left(2 S_{1}+S_{2}\right)\right]^{2}}\left(S_{0} \varepsilon_{1}-S_{1} \varepsilon_{0}\right) \cos ^{2} \theta
$$

On the 1\#2\# interface, it is expressed as (Additional file 3)

$$
P_{r 12}=-\frac{81 E^{2} R_{+}{ }^{6} S_{0}^{2}\left(S_{1}+S_{2}\right)}{2\left[2 R^{3}\left(S_{0}-S_{1}\right)\left(S_{1}-S_{2}\right)+R_{+}{ }^{3}\left(2 S_{0}+S_{1}\right)\left(2 S_{1}+S_{2}\right)\right]^{2}}\left(S_{1} \varepsilon_{2}-S_{2} \varepsilon_{1}\right) \cos ^{2} \theta
$$

Figure 3 illustrates the radial pressure distribution on both sides of the "shell" membrane. The chosen parameters are $\sigma_{0}=0.3 \mathrm{~S} / \mathrm{m}, \sigma_{2}=1.2 \mathrm{~S} / \mathrm{m}$ and $\mathrm{E}=200,000 \mathrm{~V} / \mathrm{m}$. The pressure on the outer membrane surface compresses the membrane (Fig. 3a), while the pressure on the inner membrane surface stretches and expands the membrane (Fig. $3 \mathrm{~b}$ ). On both membrane interfaces, the maximal pressure was generated on the two poles $(\theta=0$ or $\pi)$.

\section{Dependency of net radial pressure on medium conductivity}

We calculate the net pressure on the fixed membrane structure (Eq. (9), also see Additional file 4). For $\mathrm{E}=200,000 \mathrm{~V} / \mathrm{m}$, the maximal magnitude of the pressure is $3 \times 10^{4} \mathrm{~N} / \mathrm{m}^{2}$. This computed result is significant in comparison with those used for membrane deformation, including atomic force microscopy (AFM), optical trapping, micropipette aspiration and magnetic bead mocrorheology (twisting and pulling) [6].

Previous research indicates that the compressing/ pulling pressure on the cell membrane could change its 


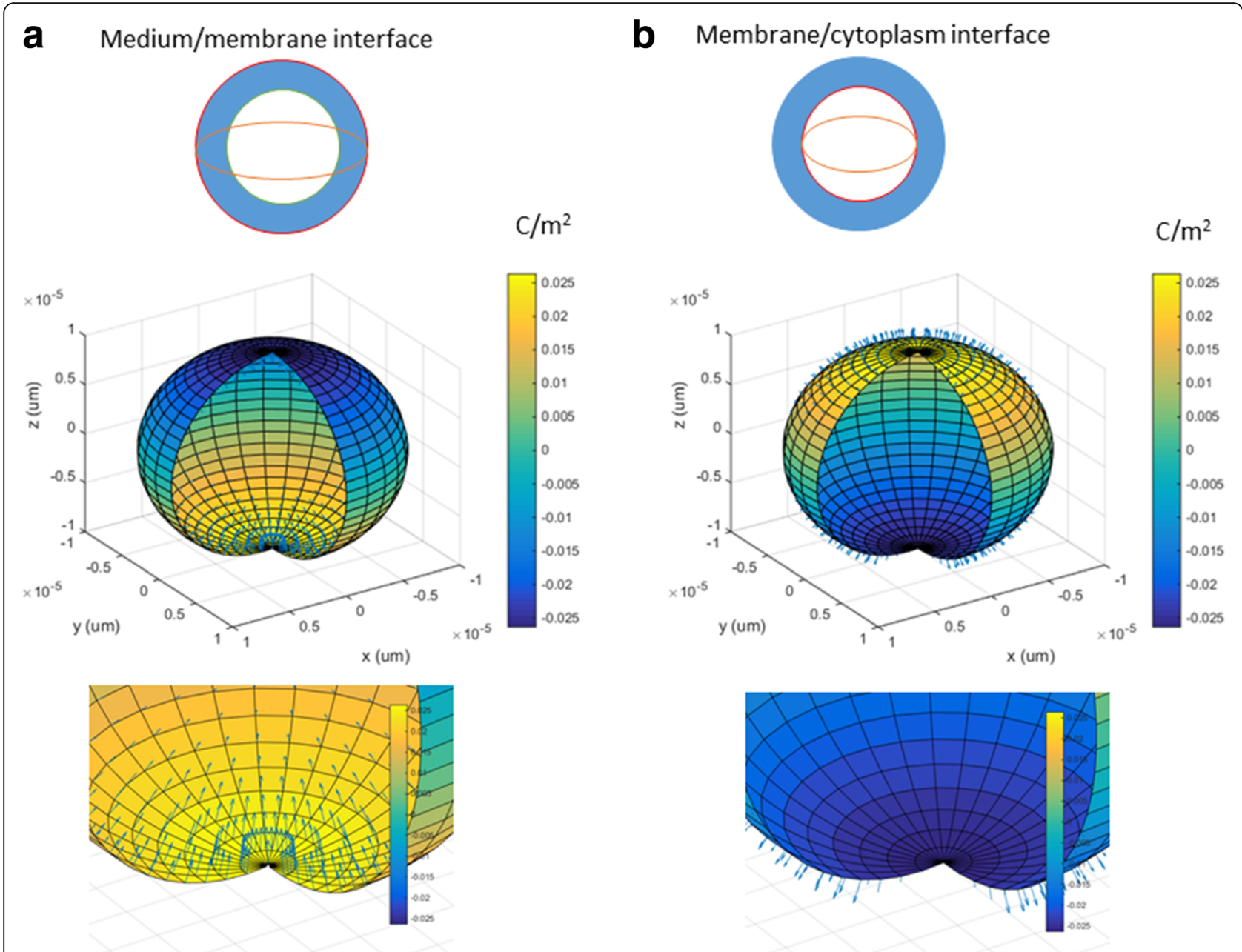

Fig. 3 Distribution of the radial pressure (arrows) on the $\mathbf{a}$ medium/membrane interface (Pr01) and on the $\mathbf{b}$ membrane/cytoplasm interface (Pr12). Color represents surface charges as defined in Fig. 2. Parameters are the same as in Fig. 2. Bottom: zoom-in views

direction, depending on the ratio between the conductivities of the medium and the cytoplasm. The pressure is pulling if the cytoplasm is more conductive and compressing if the medium is more conductive $[14,19]$. We used this observation to validate the model by varying the conductivity of the medium and computing the net radial pressure. Figure 4 illustrates that the direction of the net radial pressure switches from pulling to compressing when the medium conductivity suppresses cytoplasmic conductivity. In Fig. $4 \mathrm{a}, \sigma_{0}=0.3 \mathrm{~S} / \mathrm{m}$, $\sigma_{1}=5 \times 10^{-3} \mathrm{~S} / \mathrm{m}$ and $\sigma_{2}=1.2 \mathrm{~S} / \mathrm{m}$, the mechanic pressure pulls the membrane outwardly. In Fig. $4 \mathrm{~b}$, $\sigma_{0}=4 \mathrm{~S} / \mathrm{m}, \sigma_{1}=5 \times 10^{-3} \mathrm{~S} / \mathrm{m}$ and $\sigma_{2}=1.2 \mathrm{~S} / \mathrm{m}$, the pressure compresses the membrane.

Membrane conductivity could significantly increase in a strong electric field due to electroporation $[25,36]$. We test the effect of membrane conductivity on net radial pressure at various conductivity ratio $\left(\sigma_{0} / \sigma_{2}\right)$.
When the membrane conductivity remains low $\left(\sigma_{1}<5 \times 10^{-5} \mathrm{~S} / \mathrm{m}\right)$, the net radial pressure is always pulling, an indication of prolate elongation along the electric field. Varying medium conductivity and $\sigma_{0} / \sigma_{2}$ ratio could not cause a switch in the sign of the compressing force. In contrast, when the membrane conductivity becomes high $\left(\sigma_{1}>5 \times 10^{-4} \mathrm{~S} / \mathrm{m}\right)$, the net radial pressure can switch from pulling (when $\sigma_{0} /$ $\sigma_{2}<1$ ) to compressing (when $\sigma_{0} / \sigma_{2}>1$ ) in the field direction (Fig. 5), indicating an oblate shape.

Translational forces produced by the interaction between the induced surface charges and the local electric fields In order to calculate the forces related to the translational motion of the cell in the electric field, we need to first express the electric field in $(x, y, z)$ directions using a transformation matrix. 


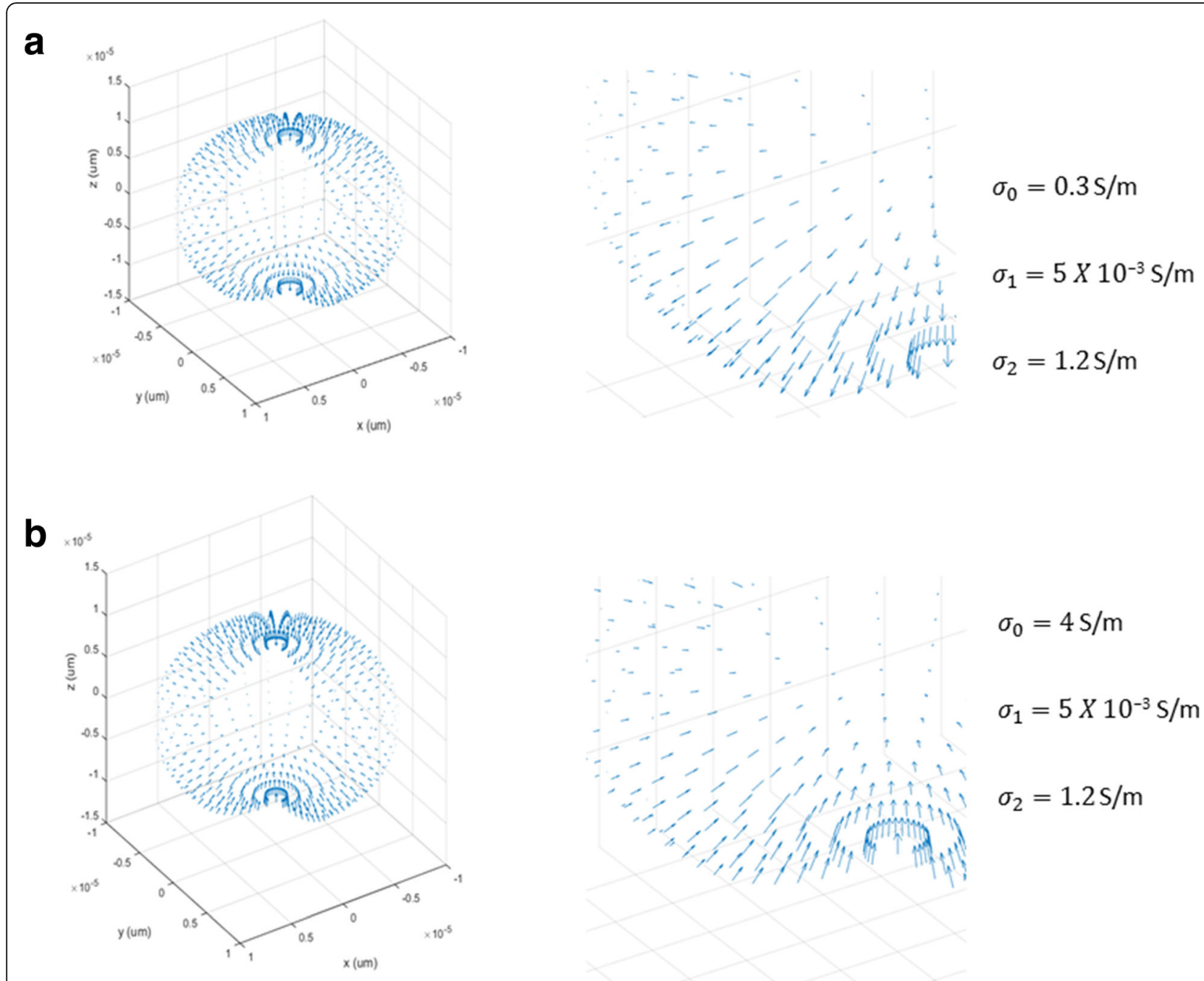

Fig. 4 Net radial pressure on the cell membrane under a strong DC electric field. a The pressure pulls the membrane along the $z$-axis when $\sigma_{0}=0.3 \mathrm{~S} / \mathrm{m}$ and $\sigma_{2}=1.2 \mathrm{~S} / \mathrm{m}$; b The pressure compresses the membrane along the z-axis when $\sigma_{0}=1.2 \mathrm{~S} / \mathrm{m}$ and $\sigma_{2}=0.3 \mathrm{~S} / \mathrm{m}$. Membrane conductivity is $\sigma_{1}=5 \times 10^{-3} \mathrm{~S} / \mathrm{m}$ in this plot

$$
\left[\begin{array}{c}
E_{o x} \\
E_{o y} \\
E_{o z}
\end{array}\right]=\left[\begin{array}{ccc}
\sin \theta \cos \phi & \cos \theta \cos \phi & -\sin \phi \\
\sin \theta \sin \phi & \cos \theta \sin \phi & \cos \phi \\
\cos \theta & -\sin \theta & 0
\end{array}\right]\left[\begin{array}{c}
E_{o r} \\
E_{o \theta} \\
E_{o \phi}
\end{array}\right]
$$

Immediately outside the cell vicinity, we obtained (Additional file 5)

$E_{0 x}=-3 E \frac{R_{-}^{3}\left(S_{0}+2 S_{1}\right)\left(S_{1}-S_{2}\right)+R_{+}^{3}\left(S_{0}-S_{1}\right)\left(2 S_{1}+S_{2}\right)}{2 R_{-}^{3}\left(S_{0}-S_{1}\right)\left(S_{1}-S_{2}\right)+R_{+}^{3}\left(2 S_{0}+S_{1}\right)\left(2 S_{1}+S_{2}\right)} \sin \theta \cos \theta \cos \phi$

$E_{0 y}=-3 E \frac{R_{-}^{3}\left(S_{0}+2 S_{1}\right)\left(S_{1}-S_{2}\right)+R_{+}^{3}\left(S_{0}-S_{1}\right)\left(2 S_{1}+S_{2}\right)}{2 R_{-}^{3}\left(S_{0}-S_{1}\right)\left(S_{1}-S_{2}\right)+R_{+}^{3}\left(2 S_{0}+S_{1}\right)\left(2 S_{1}+S_{2}\right)} \sin \theta \cos \theta \sin \phi$
$E_{0 z}=$
$3 E \frac{-S_{1}\left[2 R_{-}^{3}\left(S_{1}-S_{2}\right)-R_{+}^{3}\left(2 S_{1}+S_{2}\right)\right] \cos ^{2} \theta+S_{0}\left[R_{-}^{3}\left(S_{1}-S_{2}\right)+R_{+}^{3}\left(2 S_{1}+S_{2}\right)\right] \sin ^{2} \theta}{2 R_{-}^{3}\left(S_{0}-S_{1}\right)\left(S_{1}-S_{2}\right)+R_{+}^{3}\left(2 S_{0}+S_{1}\right)\left(2 S_{1}+S_{2}\right)}$

To test if the induced surface charges contribute to the buildup of the translational force, we integrated the translational pressure ( $x, y$ and $\mathrm{z}$ directions) on the whole spherical surface.

$$
\begin{aligned}
& F_{x_{-} \rho s}=\iint_{\theta, \phi} E_{o x} q \rho_{s 01} d a=0 \\
& F_{y \_s}=\iint_{\theta, \phi} E_{o y} q \rho_{s 01} d a=0
\end{aligned}
$$




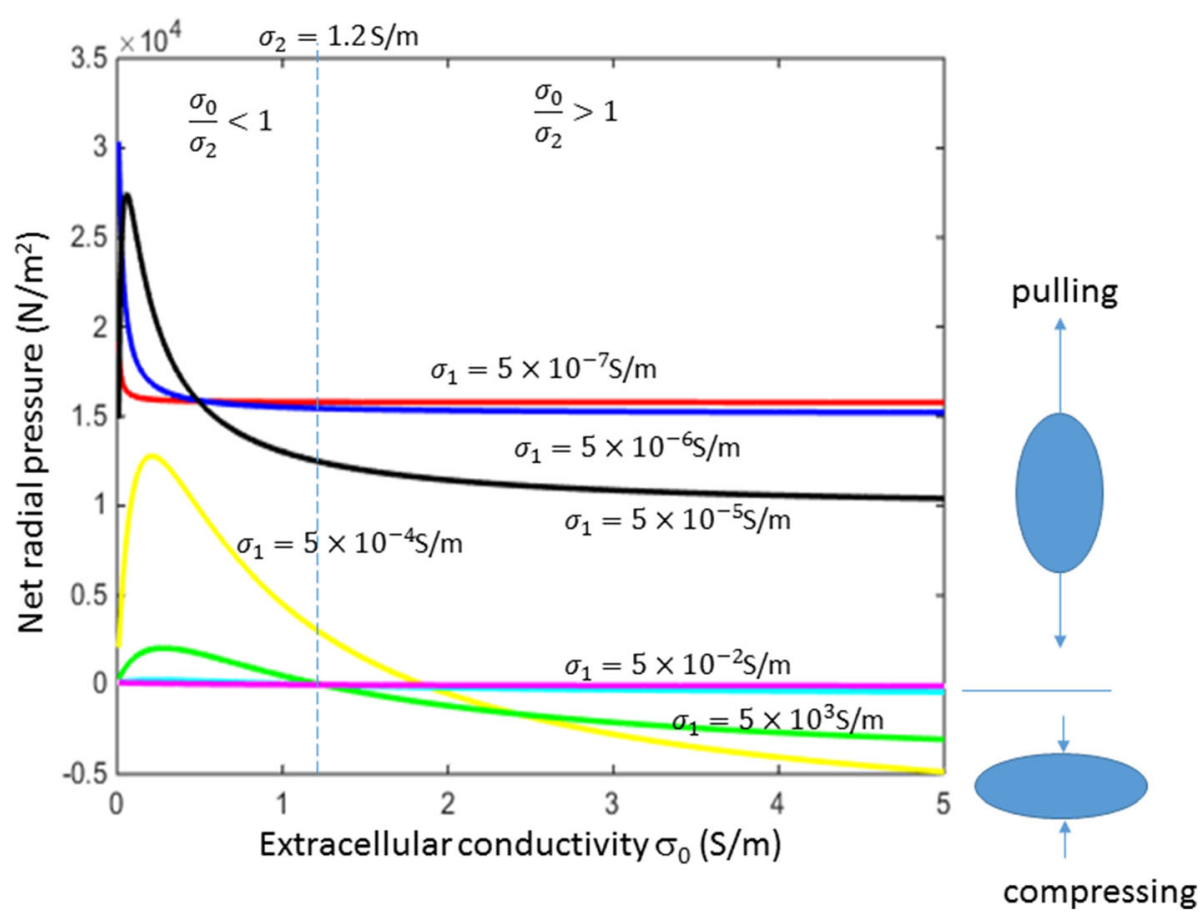

Fig. 5 Dependency of the radial pressure on the conductivities of the medium, the cytoplasm, and the membrane. When membrane conductivity $\left(\sigma_{1}\right)$ is low, the radial pressure is always pulling. When membrane conductivity is high $\left(>5 \times 10^{-4} \mathrm{~S} / \mathrm{m}\right)$, the radial pressure switches from pulling (when $\sigma_{0} / \sigma_{2}<1$ ) to compressing (when $\sigma_{0} / \sigma_{2}>1$ ). $\sigma_{2}=1.2 \mathrm{~S} / \mathrm{m}$ in this plot

$$
F_{z-\rho s}=\iint_{\theta, \phi} E_{o z} q \rho_{s 01} d a=0
$$

Here, $q$ is the elementary charge. Interaction between the induced surface charges and the local electric fields generates zero translational force. Therefore, this type of surface charge is not involved in the translational movement of the vesicle/cell inside a strong DC electric field.

\section{Translation forces generated by the interaction between} the electric field and the intrinsic charges

Under normal physiological conditions, cell membrane is non-neutral due to the presence of the charged lipid head groups embedded inside the membrane [37]. The accumulation of anionic phospholipids (i.e., phosphatidylserine and phosphatidylinositol) produces an intensive electric field $\left(10^{5} \mathrm{~V} / \mathrm{cm}\right)$, which can strongly attract ions, cationic proteins and peptides $[38,39]$. This is a biophysics mechanism essential for protein targeting and intracellular signaling. Translation forces (Additional file 6) can be generated for a biological cell when the electric field interacts with the intrinsic surface charges (designated as $\rho_{p}$ ).

$$
\begin{aligned}
F_{x_{-} \rho_{p}} & =\iint_{\theta, \phi} E_{o x} \rho_{p} q d a \\
& =\iint_{\theta, \phi}\left[\begin{array}{l}
-3 E \frac{R_{-}^{3}\left(S_{0}+2 S_{1}\right)\left(S_{1}-S_{2}\right)+R_{+}^{3}\left(S_{0}-S_{1}\right)\left(2 S_{1}+S_{2}\right)}{2 R_{-}^{3}\left(S_{0}-S_{1}\right)\left(S_{1}-S_{2}\right)+R_{+}^{3}\left(2 S_{0}+S_{1}\right)\left(2 S_{1}+S_{2}\right)} \\
\quad
\end{array}\right. \\
& =0
\end{aligned}
$$

$$
\begin{aligned}
F_{y_{-} \rho_{p}} & =\iint_{\theta, \phi} E_{o x} \rho_{p} q d a \\
& =\iint_{\theta, \phi}\left[\begin{array}{l}
-3 E \frac{R_{-}^{3}\left(S_{0}+2 S_{1}\right)\left(S_{1}-S_{2}\right)+R_{+}^{3}\left(S_{0}-S_{1}\right)\left(2 S_{1}+S_{2}\right)}{2 R_{-}^{3}\left(S_{0}-S_{1}\right)\left(S_{1}-S_{2}\right)+R_{+}^{3}\left(2 S_{0}+S_{1}\right)\left(2 S_{1}+S_{2}\right)} \\
\quad \sin \theta \cos \theta \sin \phi] \rho_{p} q R_{+}^{2} \sin \theta d \theta d \phi
\end{array}\right. \\
& =0
\end{aligned}
$$

$$
\begin{aligned}
F_{z_{-} \rho_{p}} & =\iint_{\theta, \phi} E_{o z} \rho_{p} q d a \\
& =\iint_{\theta, \phi}\left[3 E \frac{-S_{1}\left[2 R_{-}^{3}\left(S_{1}-S_{2}\right)-R_{+}^{3}\left(2 S_{1}+S_{2}\right)\right] \cos ^{2} \theta+S_{0}\left[R_{-}^{3}\left(S_{1}-S_{2}\right)+R_{+}^{3}\left(2 S_{1}+S_{2}\right)\right] \sin ^{2} \theta}{2 R_{-}^{3}\left(S_{0}-S_{1}\right)\left(S_{1}-S_{2}\right)+R_{+}^{3}\left(2 S_{0}+S_{1}\right)\left(2 S_{1}+S_{2}\right)}\right] \rho_{p} q R_{+}^{2} \sin \theta d \theta d \phi \\
& =4 \pi R_{+}^{2} \rho_{p} E=Q_{p} E
\end{aligned}
$$


Therefore, translational force generated on a cell is proportional to the net intrinsic charge carried by the cell itself $\left(Q_{p}\right)$, as suggested previously [40]. If we assume the intrinsic charge density to be $3.6 \times 10^{4}$ electronic charges/square micron [8] and cell radius to be $10 \mu \mathrm{m}$, we obtain $F_{z \rho_{p}}=1.6 \times 10^{-6} \mathrm{~N}$, a force comparable to that generated by migrating or contracting cells $\left(10^{-9} \mathrm{~N}\right.$ $10^{-5} \mathrm{~N}$ in [6]).

\section{Discussion}

Understanding vesicle biomechanics under electric field exposure brings valuable insights into the behavior of biological cells, since both share the same structure components, the enclosing bilipid membrane. However, the overall kinematics of the vesicles and cells are different in a strong DC electric field. Vesicles demonstrate deformation in the strong DC electric field, while cells demonstrate both deformation and migration.

This work provides a three-dimensional modeling of the interaction between a strong DC electric field and the low-conductive, capacitive shell membrane of a cell/vesicle. It provides a set of analytical solutions for the surface charges and electric field distributions. It also provides mechanical analyses on the fixed membrane surface, including the radial pressure for membrane deformation and the translational forces for movement. The distribution of the induced surface charges depends on the orientation of the electric field to the structure. When the membrane becomes significantly conductive in the strong electric field, the directionality of the radial pressure on the membrane can switch between pulling and compressing, depending on the cytoplasm/medium conductivity ratio. Finally, translational force can be built on the structure for migration via its interaction with the intrinsic charges on the membrane, rather than with the induced electric charges. The model thus reconciles the kinematic difference between a cell (deformation and migration) and a vesicle (deformation only) under a strong DC electric field.

\section{Surface charges induced by the externally applied field on the membrane}

Analytical expressions were given for the induced surface charges on both sides of the membrane in a DC electric field. On a single interface, the charges are separate by their polarities, making the spherical interface an equivalent electric dipole (Fig. 2, also see [41]). For a $200,000 \mathrm{~V} / \mathrm{m}$ electric field, the surface charges on the poles can reach $0.025 \mathrm{C} / \mathrm{m}^{2}$, a value that is several folds greater than those charges carried by the cell itself (i.e., $5.76 \times 10^{-3} \mathrm{C} / \mathrm{m}^{2}$ in isolated toad bladder epithelial cells in [8]). The biological effects of such charge accumulation can include an extra voltage to be superimposed on the membrane [25, 26, 42], alteration of meta-stable membrane structure [43], or electroporation of the membrane [44]. More importantly, membrane surface charges are essential in producing electric compressive force for membrane deformation [22]. The static radial pressure on the membrane surface is produced by the interaction between the local electric field and the induced surface charges [45], which can be a complicated function of both the electric/geometrical properties of the shell and the properties of the field (i.e., Eqs. (14.1) and (14.2)).

\section{Impact of conductivity ratio on net radial pressure}

Experimentally, it has been shown that vesicle deformation inside an electric field is dependent on the conductivities of both the suspending fluid and the interior cytoplasm [46, 47]. Our model supports this observation, since increasing the medium conductivity could induce the switching of the radial pressure from pulling (Fig. 4a) to compressing (Fig. 4b). Furthermore, this conductivity ratio-dependent switch can only happen when the membrane conductivity is significantly increased (Fig. 5). Considering that the field intensity is $200,000 \mathrm{~V} / \mathrm{m}$ in this study, a value that is sufficient for vesicle deformation [15] and membrane poration [16], it is likely the membrane conductivity could increase prominently.

Previous works have demonstrated that membrane conductance plays a significant role in inducing transmembrane potential and vesicle shape transition. Using a zero-thickness model of membrane and a hybrid numerical (immersed boundary and immersed interface) method, $\mathrm{Hu}$ et al. [22] found that increase in the membrane conductance is required for the vesicle to remain oblate shape in a DC electric field. Using a similar zerothickness and numerical simulation, McConnell et al. [23] found that the vesicle only experiences oblate steady deformation when $\sigma_{0} / \sigma_{2}>1$ and membrane conductance is high. In these zero-thickness membrane model, transmembrane potential is calculated as a "jump" of potential, and the boundary condition of potential continuity across the two media is invalid. Our work uses a more biologically relevant representation of the "shelllike" membrane (approximately $5 \mathrm{~nm}$ in thickness), since the physical features (i.e., thickness and radius) of the membrane plays a significant role in electric field distribution, membrane polarization [45], and membrane biomechanics under electromagnetic field [32, 33]. Therefore, the "shell" model not only allows potential continuity across the membrane surface (boundary condition 1), but also allows the calculation of the membrane charges on both side of the membrane. Our analytical solutions support these previous modeling 
results. When $\sigma_{0} / \sigma_{2}<1$, the radial pressure is consistently pulling (prolate), regardless if the membrane conductivity is high. In contrast, when $\sigma_{0} / \sigma_{2}>1$, the radial pressure is pulling when the membrane conductance is low, and compressing (oblate) when membrane conductance is high $\left(\sigma_{1}>5 \times 10^{-4} \mathrm{~S} / \mathrm{m}\right)$.

\section{Differential migration kinematics between a vesicle and a cell}

Our model is successful in reconciling the kinematic difference between a spherical vesicle and a cell with similar shape in a strong DC electric field.

We found that interaction between the induced surface charges and local electric fields produces minimal translational force (Eq. 19.1 to 19.3). Using a method that treated the polarized particle as a dipole, Jones also ound that particles should experience zero net translation force in an even DC field [41] if only the induced electric charges are considered. This could easily be the case for an artificial vesicle, which is made with neutral molecules (L-a-phosphatidylcholine [17]) and its intrinsic surface charges are assumed to be zero. For a biological cell, intrinsic charges is regarded important in its cathodal galvanotaxis [48]. In a surprising simple form, we found the translational force is proportional to the overall net charge carried by the cell (Eq. 20.3). It is reasonable to assume that an evenly distributed intrinsic charge pattern on the cell membrane could produce a zero net radial pressure (Eq. 9). Therefore, the intrinsic charges could contribute to the translational motion of the cell, but not membrane deformation.

The model provides several interesting hypotheses regarding vesicle and cell kinematics. First, since the intrinsic charges are only associated with cell migration (but not membrane deformation) in the strong DC electric field, manipulations of these intrinsic charges should change cell mobility (but not deformation) in the field. Methods includes changing $\mathrm{pH}$ value [40] and applying calcium or magnesium to bind the surface charges [8]. Second, partially charged vesicles could be made to mimic cell migrations in the strong DC electric field. These vesicles could be made with lipid mixtures of phosphatidylcholine and phosphatidylglycerol or phosphatidylcholine and phosphatidylserine [15].

It is possible that the distribution of the intrinsic charge will be altered by the electric field [49]. However, if the polarity of the net charge $\left(Q_{p}\right)$ is not affected by their redistribution, direction of the translations force should not be altered (Eq. 20.3). In supporting this model prediction, it was found that the re-distribution of the charged cell surface proteins did not alter the direction of galvanotaxis [49].

It shall be noted that if the intrinsic surface proteins are geometrically inhomogeneous, they may play an important role in cell deformation under a DC field exposure. For example, interactions between the charged protein on the outer hair cells and a transcellular oscillating electric field can cause cell vibration, leading to the deformation of the cell [50]. If one knows the detailed distribution of the charged surface proteins, it is possible to use Eq. (8) to deduce the local pressure generated by the intrinsic charges.

\section{Future directions}

Several assumptions have been made to formulate the present model for computational simplicity. First, the model does not represent the geometrical complexity in a real biological cell. Second, the model assumes both the medium and cytoplasmic environment are homogeneous. While this may be the case for vesicles, it could be unrealistic for cells $[51,52]$. Third, the model does not consider mechanical factors such as hydrodynamic pressure around the structure [14] and membrane elasticity [4]. Finally, membrane polarization [53] in the strong DC electric field allows for calcium influx, which could alter membrane shape via actin polymerization/ depolymerization and actomyosin contractility [54]. Electroporation could also alter local electric fields [55] by allowing ion exchange through the pores, which in turn alter vesicle shapes. Future work should consider multi-compartment modeling or finite element meshes [56-58] to represent the detailed anatomic complexity of a biological cell. More complicated simulation should consider numerical methods, whose accuracy is subjected to be validated by analytical works including this one.

\section{Conclusions}

The paper studies cellular biomechanics under a strong DC electric field exposure, by simulating the membrane with a "shell-like" structure. It provides the analytical solutions for the pressure and translational force that might contribute to the "shell" deformation and movement, respectively. The model provides a quantitative interpretation for various vesicle deformation scenarios inside the medium with different conductivities. It also explains the kinematic differences between a vesicle and a spherical cell inside the strong DC electric field.

\section{Additional files}

Additional file 1: Basic equations. (PDF $92 \mathrm{~kb}$ )

Additional file 2: Interface01. (PDF $115 \mathrm{~kb}$ )

Additional file 3: Interface12. (PDF $112 \mathrm{~kb}$ )

Additional file 4: Net radial pressure. (PDF $51 \mathrm{~kb}$ )

Additional file 5: Translation force -induced charges. (PDF $65 \mathrm{~kb}$ )

Additional file 6: Translation force- intrinsic charges. (PDF $48 \mathrm{~kb}$ ) 


\section{Abbreviations}

$F_{x \rho_{s}}, F_{y \rho_{s}}, F_{y \rho_{s}}$ : Translational force $(\mathrm{N})$ applied on the membrane in the Cartesian coordinate system $(\vec{x}, \vec{y}, \vec{z})$ due to the interaction between induced surface charges and the local electric field; $F_{x \rho_{p}}, F_{y \rho_{0}}, F_{y \rho_{0}}$ : Translational force $(\mathrm{N})$ on the membrane in the Cartesian coordinate system $(\vec{x}, \vec{y}, \vec{z})$, due to the interaction between the intrinsic membrane charges and the local electric field; $E$ : Intensity of externally-applied electric field $(\mathrm{V} / \mathrm{m})$; $E_{0 r,} E_{0 \theta}, E_{0 \varphi}$ : Electric field intensity in the medium $(\mathrm{V} / \mathrm{m})$ in the spherical coordinate system $(\vec{r}, \vec{\theta}, \vec{\varphi}) ; E_{0 x,} E_{0 y,} E_{0 z:}$ Electric field intensity in the medium $(\mathrm{V} / \mathrm{m})$ in the Cartesian coordinate system $(\vec{x}, \vec{y}, \vec{z}) ; E_{1 r} E_{1 \theta}$ $E_{1 \varphi}$ : Electric field intensity in the membrane $(\mathrm{V} / \mathrm{m})$ in the spherical coordinate system $(\vec{r}, \vec{\theta}, \vec{\varphi}) ; E_{1 x,} E_{2 y \prime} E_{1 z}$ : Electric field intensity inside the membrane $(\mathrm{V} / \mathrm{m})$ in the Cartesian coordinate system $(\vec{x}, \vec{y}, \vec{z}) ; E_{2 r}, E_{2 \theta_{r}}$ $E_{2 \varphi}$ : Electric field intensity in the cytoplasm $(\mathrm{V} / \mathrm{m})$ in the spherical coordinate system $(\vec{r}, \vec{\theta}, \vec{\varphi}) ; E_{2 x}, E_{2 y}$, $E_{2 z}$ : Electric field intensity in the cytoplasm $(\mathrm{V} / \mathrm{m})$ in the Cartesian coordinate system $(\vec{x}, \vec{y}, \vec{z}) ; P_{r}$ : Net radial pressure on the membrane $\left(\mathrm{N} / \mathrm{m}^{2}\right)_{\text {; }} P_{512}$ : Radial pressure on the membrane/cytoplasm interface $\left(\mathrm{N} / \mathrm{m}^{2}\right)$; $q$ : Elementary charge, $\mathrm{q}=1.60217662 \times 10^{-19} \mathrm{C}_{;} \mathrm{Q}_{501}$ : Amount of net induced surface charges on the medium/membrane interface $(C) ; Q_{501}$ : Radial pressure on the medium/membrane interface $\left(\mathrm{N} / \mathrm{m}^{2}\right) ; Q_{s 12}$ : Amount of net induced surface charges on the membrane/ cytoplasm interface $(C) ; \rho_{p}$ : Intrinsic charge density on the cell membrane $\left(\mathrm{C} / \mathrm{m}^{2}\right) ; \rho_{501}$ : Induced surface charge density on the medium/membrane interface $\left(\mathrm{C} / \mathrm{m}^{2}\right) ; \rho_{s 12}$ : Induced surface charge density on the membrane/ cytoplasm interface $\left(\mathrm{C} / \mathrm{m}^{2}\right)$

\section{Acknowledgements}

Stephanie Kaszuba assisted with editing of the manuscript.

\section{Funding}

The author thanks the Research Support Grant from Loyola University Chicago.

\section{Availability of data and materials}

All data generated or analyzed during this study are included in this published article [and its Additional files.]

\section{Authors' contributions}

HY formulated the model, solved the mathematical equations, ran the simulation, and drafted the manuscript.

\section{Ethics approval and consent to participate}

Not applicable.

\section{Consent for publication}

Not applicable.

\section{Competing interests}

The author declares that he has no competing interests.

\section{Publisher's Note}

Springer Nature remains neutral with regard to jurisdictional claims in published maps and institutional affiliations.

Received: 7 March 2017 Accepted: 17 August 2017

\section{Published online: 25 August 2017}

\section{References}

1. Sens $P$, Isambert $H$. Undulation instability of lipid membranes under an electric field. Phys Rev Lett. 2002;88(12):128102.

2. Bae C, Butler PJ. Finite element analysis of microelectrotension of cell membranes. Biomech Model Mechanobiol. 2008;7:379-86.

3. Bryant $\mathrm{G}$, Wolfe J. Electromechanical stresses produced in the plasma membranes of suspended cells by applied electric fields. J Membr Biol. 1987:96:129-39.

4. Engelhardt H, Sackmann E. On the measurement of shear elastic moduli and viscosities of erythrocyte plasma membranes by transient deformation in high frequency electric fields. Biophys J. 1988:54:495-508.

5. Tandon N, Goh B, Marsano A, Chao PH, Montouri-Sorrentino C, Gimble J, Vunjak-Novakovic G. Alignment and elongation of human adipose-derived stem cells in response to direct-current electrical stimulation. Conf Proc IEEE Eng Med Biol Soc. 2009;2009:6517-21.
6. Kamm R, Lammerding J, Mofrad M. In: Bhushan B, editor. Cellular Nanomechanics, Springer Handbook of Nanotechnology; 2010.

7. Coulter CB. The Isoelectric Point of Red Blood Cells and Its Relation to Agglutination. The Journal of general physiology. 1921;3:309-23.

8. Lipman KM, Dodelson R, Hays RM. The surface charge of isolated toad bladder epithelial cells. Mobility, effect of $\mathrm{pH}$ and divalent ions. The Journal of general physiology. 1966;49:501-16.

9. Korohoda W, Wilk A. Cell electrophoresis-a method for cell separation and research into cell surface properties. Cell Mol Biol Lett. 2008;13:312-26.

10. Koppes AN, Zaccor NW, Rivet CJ, Williams LA, Piselli JM, Gilbert RJ, Thompson DM. Neurite outgrowth on electrospun PLLA fibers is enhanced by exogenous electrical stimulation. J Neural Eng. 2014;11:046002.

11. Koppes AN, Nordberg AL, Paolillo GM, Goodsell NM, Darwish HA, Zhang L, Thompson DM. Electrical stimulation of schwann cells promotes sustained increases in neurite outgrowth. Tiss Eng A. 2014:20:494-506.

12. Meng X, Arocena M, Penninger J, Gage FH, Zhao M, Song B. PI3K mediated electrotaxis of embryonic and adult neural progenitor cells in the presence of growth factors. Exp Neurol. 2011;227:210-7.

13. Jeong SH, Jun SB, Song JK, Kim SJ. Activity-dependent neuronal cell migration induced by electrical stimulation. Med Biol Eng Comput. 2009:47:93-9.

14. Vlahovska PM, Gracia RS, Aranda-Espinoza S, Dimova R. Electrohydrodynamic model of vesicle deformation in alternating electric fields. Biophys J. 2009;96:4789-803.

15. Riske KA, Dimova R. Electric pulses induce cylindrical deformations on giant vesicles in salt solutions. Biophys J. 2006;91:1778-86.

16. Sadik MM, Li J, Shan JW, Shreiber DI, Lin H. Vesicle deformation and poration under strong dc electric fields. Phys Rev E Stat Nonlinear Soft Matter Phys. 2011;83:066316.

17. Pasenkiewicz-Gierula M, Takaoka Y, Miyagawa H, Kitamura K, Kusumi A. Charge pairing of headgroups in phosphatidylcholine membranes: A molecular dynamics simulation study. Biophys J. 1999;76:1228-40.

18. Riske KA, Dimova R. Electro-deformation and poration of giant vesicles viewed with high temporal resolution. Biophys J. 2005;88:1143-55.

19. Hyuga H, Kinosita K, Wakabayashi N. Deformation of Vesicles under the Influence of Strong Electric-Fields II. Jpn J Appl Phys Part 1. 1991;30:1333-5.

20. Hyuga $H$, Kinosita K, Wakabayashi N. Deformation of Vesicles under the Influence of Strong Electric-Fields. Jpn J Appl Phys Part 1. 1991;30:1141-8,

21. Veerapaneni $S$. Integral equation methods for vesicle electrohydrodynamics in three dimensions. J Comput Phys. 2016;326:278-89.

22. Hu WF, Lai MC, Seol Y, Young YN. Vesicle electrohydrodynamic simulations by coupling immersed boundary and immersed interface method. J Comput Phys. 2016;317:66-81.

23. McConnell LC, Vlahovska PM, Miksis MJ. Vesicle dynamics in uniform electric fields: squaring and breathing. Soft Matter. 2015;11:4840-6.

24. Kotnik T, Miklavcic D. Second-order model of membrane electric field induced by alternating external electric fields. IEEE Trans Biomed Eng. 2000;47:1074-81.

25. Mossop BJ, Barr RC, Henshaw JW, Yuan F. Electric fields around and within single cells during electroporation-a model study. Ann Biomed Eng. 2007;35:1264-75

26. Ye H, Cotic M, Carlen PL. Transmembrane potential induced in a spherical cell model under low-frequency magnetic stimulation. J Neural Eng. 2007;4:283-93.

27. Kotnik T, Bobanovic F, Miklavcic D. Sensitivity of transmembrane voltage induced by applied electric fields - a theoretical analysis. Bioelectrochem Bioenerg. 1997:43:285-91.

28. Zhang ZJ, Koifman J, Shin DS, Ye H, Florez CM, Zhang L, Valiante TA, Carlen PL. Transition to seizure: ictal discharge is preceded by exhausted presynaptic GABA release in the hippocampal CA3 region. The Journal of neuroscience: the official journal of the Society for Neuroscience. 2012;32:2499-512

29. Griffiths DJ. Introduction to Electrodynamics. 3rd ed; 1999.

30. Kotnik T, Miklavcic D. Analytical description of transmembrane voltage induced by electric fields on spheroidal cells. Biophys J. 2000;79:670-9.

31. Polk C, Song JH. Electric fields induced by low frequency magnetic fields in inhomogeneous biological structures that are surrounded by an electric insulator. Bioelectromagnetics. 1990;11:235-49.

32. Ye H, Curcuru A. Vesicle biomechanics in a time-varying magnetic field. BMC Biophys. 2015;8:2.

33. Ye H, Curcuru A. Biomechanics of cell membrane under low-frequency time-varying magnetic field: a shell model. Med Biol Eng Comput. 2016;54:1871-81. 
34. Zhang PC, Keleshian AM, Sachs F. Voltage-induced membrane movement Nature. 2001;413:428-32.

35. Voldman J. Electrical forces for microscale cell manipulation. Annu Rev Biomed Eng. 2006:8:425-54.

36. Mossop BJ, Barr RC, Zaharoff DA, Yuan F. Electric fields within cells as a function of membrane resistivity-a model study. IEEE Trans Nanobioscience. 2004;3:225-31.

37. Goldenberg NM, Steinberg BE. Surface charge: a key determinant of protein localization and function. Cancer Res. 2010;70:1277-80.

38. McLaughlin S. The electrostatic properties of membranes. Annu Rev Biophys Biophys Chem. 1989;18:113-36.

39. Olivotto M, Arcangeli A, Carla M, Wanke E. Electric fields at the plasma membrane level: A neglected element in the mechanisms of cell signalling. BioEssays. 1996;18:495-504.

40. Mehrishi JN, Bauer J. Electrophoresis of cells and the biological relevance of surface charge. Electrophoresis. 2002;23:1984-94.

41. Jones TB. Electromechanics of particles. Cambridge: Cambridge University Press; 1995.

42. Pucihar G, Miklavcic D, Kotnik T. A time-dependent numerical model of transmembrane voltage inducement and electroporation of irregularly shaped cells. IEEE Trans Biomed Eng. 2009;56:1491-501.

43. Sukharev SI, Klenchin VA, Serov SM, Chernomordik LV, Chizmadzhev Yu A. Electroporation and electrophoretic DNA transfer into cells. The effect of DNA interaction with electropores. Biophys J. 1992;63:1320-7.

44. Kinosita K Jr, Tsong TY. Voltage-induced pore formation and hemolysis of human erythrocytes. Biochim Biophys Acta. 1977:471:227-42.

45. Ye H, Steiger A. Neuron matters: electric activation of neuronal tissue is dependent on the interaction between the neuron and the electric field. J Neuroeng Rehabil. 2015;12:65.

46. Dimova R, Riske KA, Aranda S, Bezlyepkina N, Knorr RL, Lipowsky R. Giant vesicles in electric fields. Soft Matter. 2007;3:817-27.

47. Aranda S, Riske KA, Lipowsky R, Dimova R. Morphological transitions of vesicles induced by alternating electric fields. Biophys J. 2008:95:L19-21.

48. Djamgoz MBA, Mycielska M, Madeja Z, Fraser SP, Korohoda W. Directional movement of rat prostate cancer cells in direct-current electric field: involvement of voltage-gated Na+ channel activity. J Cell Sci. 2001;114:2697-705.

49. Finkelstein El, Chao PH, Hung CT, Bulinski JC. Electric field-induced polarization of charged cell surface proteins does not determine the direction of galvanotaxis. Cell Motil Cytoskeleton. 2007;64:833-46.

50. Jen DH, Steele CR. Electrokinetic model of cochlear hair cell motility. J Acoust Soc Am. 1987;82:1667-78.

51. Holsheimer J. Electrical conductivity of the hippocampal CA1 layers and application to current-source-density analysis. Exp Brain Res. 1987;67:402-10.

52. Tyner KM, Kopelman R, Philbert MA. "Nanosized voltmeter" enables cellularwide electric field mapping. Biophys J. 2007;93:1163-74.

53. Gao RC, Zhang XD, Sun YH, Kamimura Y, Mogilner A, Devreotes PN, Zhao M. Different roles of membrane potentials in electrotaxis and chemotaxis of dictyostelium cells. Eukaryot Cell. 2011;10:1251-6.

54. Mycielska ME, Djamgoz MBA. Cellular mechanisms of direct-current electric field effects: galvanotaxis and metastatic disease. J Cell Sci. 2004;117:1631-9.

55. Mossop BJ, Barr RC, Henshaw JW, Yuan F. Electric fields around and within single cells during electroporation - A model study. Ann Biomed Eng. 2007;35:1264-75.

56. Rattay F. Analysis of the electrical excitation of CNS neurons. IEEE Trans Biomed Eng. 1998;45:766-72.

57. McIntyre CC, Grill WM, Sherman DL, Thakor NV. Cellular effects of deep brain stimulation: model-based analysis of activation and inhibition. J Neurophysiol. 2004;91:1457-69.

58. Pucihar G, Kotnik T, Valic B, Miklavcic D. Numerical determination of transmembrane voltage induced on irregularly shaped cells. Ann Biomed Eng. 2006:34:642-52.

\section{Submit your next manuscript to BioMed Central and we will help you at every step:}

- We accept pre-submission inquiries

- Our selector tool helps you to find the most relevant journal

- We provide round the clock customer support

- Convenient online submission

- Thorough peer review

- Inclusion in PubMed and all major indexing services

- Maximum visibility for your research

Submit your manuscript at www.biomedcentral.com/submit
Biomed Central 